\author{
Ana Lalic ${ }^{*}$ \\ Università di Sarajevo
}

\title{
LA REALIZZAZIONE DEGLI ATTI LINGUISTICI NEI TESTAMENTI MEDIEVALI
}

\begin{abstract}
Nel presente contributo ci proponiamo di analizzare, attraverso la lente della pragmatica storica, $\mathrm{i}$ tipi di atti linguistici in un corpus di testamenti italiani redatti a Ragusa verso la fine del Trecento e durante il Quattrocento. L'obiettivo del nostro lavoro è individuare gli atti linguistici corrispondenti alla forma del testamento, quelli direttamente determinati da essa o dal contesto di provenienza. A tal fine riprendiamo il quadro teorico di Austin (1962) nel tentativo di applicarlo alla diacronia. L'ipotesi primaria della ricerca è che la maggior parte degli atti linguistici provenga dalla forma del testamento e dalla sua natura giuridica e che possa essere riconosciuta dalla struttura grammaticale. I risultati della ricerca implicano che la teoria di Austin possa essere impiegata nelle ricerche diacroniche e che gli atti linguistici dei testamenti siano determinati dalla forma convenzionale del testamento come atto giuridico.

Parole chiave: testamenti, atti giuridici, atti linguistici, pragmatica, grammatica, diacronia.
\end{abstract}

\section{INTRODUZIONE}

L'idea che "facciamo" cose usando le parole è una nozione accettata e presente nel pensiero linguistico almeno a partire da Austin (1962) nel suo contributo epocale How To Do Things with Words (tradotto in italiano con il titolo Come fare cose con le parole). La pragmatica storica, cioè l'idea che la teoria di Austin possa essere applicata anche in diacronia, compie un importante passo avanti con Jucker (2000) e negli ultimi decenni si è affermata come ambito rilevante della linguistica storica. Tenteremo di collocare questo contributo nel sempre più crescente settore delle ricerche sulla pragmatica storica, sugli usi e sugli schemi comunicativi del passato.

In questa ricerca cercheremo di applicare la teoria moderna degli atti linguistici a un corpus di testamenti medievali. A tal fine il lavoro è diviso

\footnotetext{
*ana.lalic@ff.unsa.ba
} 
in due parti: la prima offrirà un'elaborazione teorica, mentre la seconda sarà dedicata all'analisi empirica del corpus. Anzitutto si focalizza la questione metodologica delle ricerche sulla pragmatica diacronica e sulle modalità di applicazione della teoria degli atti linguistici a un corpus di epoca medievale. Secondariamente si procede con la selezione dei testamenti che costituiranno il corpus e con la descrizione del corpus così formato.

Infine, si costruisce il quadro teorico degli atti linguistici che sarà applicato all'analisi del corpus. Per quanto riguarda l'analisi empirica del corpus, il commento degli atti linguistici mira a mostrare le caratteristiche primarie di ogni atto individuato, le modalità della sua individuazione e quali strategie sono state adoperate nel determinarlo.

Le principali domande alle quali cercheremo di rispondere sono se la teoria di Austin possa essere applicata diacronicamente, se la grammatica influisca sulla forma del testamento, quali elementi grammaticali siano importanti nella determinazione di un atto linguistico, se la forma del testamento influisca sulla scelta dell'atto linguistico e come la grammatica e il contesto possano cambiare la comprensione del messaggio.

\section{LA QUESTIONE METODOLOGICA}

La pragmatica storica mira a descrivere e a comprendere le convenzioni dell'uso della lingua in comunità estinte che non possono essere osservate direttamente (Jacobs \& Jucker 1995: 6), il che rende necessaria la selezione accurata del corpus da analizzare. Poiché la pragmatica tratta per lo più la lingua viva e il dialogo (Jucker 2000), nel campo della pragmatica storica si pone il problema di vagliare corpus congrui considerando la mancanza di materiali idonei, che riportino in modo autentico la lingua parlata nelle epoche passate.

La questione metodologica principale della pragmatica storica riguarda la determinazione del grado di approssimazione della lingua trascritta al registro orale dell'epoca (Jacobs \& Jucker 1995; Jucker 2000; Kohnen 2007; Kryk-Kastovsky 2009). Jucker (2000) individua anche un secondo problema, quello della variazione diacronica, ovvero il fatto che un atto linguistico realizzato in lingue moderne non deve corrispondere a un atto del passato. Kryk-Kastovsky (2009), eppure, afferma che esistono corrispondenze fra la lingua usata oggi e quella del passato, e che un metodo moderno usato nelle ricerche sincroniche, come quello degli atti linguistici, può essere applicato anche alla diacronia.

Tuttavia, l'aspetto della diacronia è stato a lungo trascurato nelle ricerche sugli atti linguistici. Negli ultimi anni sono stati pubblicati alcuni lavori importanti, la maggior parte in lingua inglese (per esempio Arnovick 1999; 
Jucker 2000; Kohnen 2007; Kohnen 2008a; Kohnen 2008b; Kryk-Kastovsky 2009; King 2011). Inoltre, la mera quantità delle ricerche pubblicate dal 1995 (alcune già citate) e l'esistenza del Journal of Historical Pragmatics mostrano lo sviluppo del campo di indagine scientifica e indicano che la metodologia sincronica può essere applicata alla diacronia: un punto di vista che riprendiamo per la nostra ricerca.

Nello specifico, Bertucelli Papi (2000) conclude che le ricerche diacroniche eseguite con il modello sincronico sono possibili se si prendono in considerazione il contesto storico, il cambiamento semantico e le differenze sociologiche. La forma del testamento risulta adeguata a superare questo limite in quanto formulaico e poiché la sua struttura fissa non permette interpretazioni soggettive. I testi legali sono peculiari nella loro forma perché sono di carattere performativo (Visconti 2009) e presentano una situazione di monologo (Jucker 2000) giacché non viene stabilito un rapporto comunicativo fra due parlanti e non è prevista una risposta dell'interlocutore. Alcuni autori (Kurzon 1986; Bach 1995; Visconti 2009; Nakaš 2010; Tollerton 2011; Lovrić Jović 2006, 2013) basano le indagini sulla lingua viva e parlata delle epoche passate proprio sugli atti legali e i testamenti, ipotizzando che questi rappresentino la lingua viva. In questi testi infatti, a differenza delle opere letterarie, l'autore non è vincolato dalle esigenze stilistiche e letterarie e può esprimersi utilizzando la lingua autentica (Bartoli 2000, Lovrić Jović 2006). Si è inoltre evinto che il carattere formulaico del testamento mette in evidenza la struttura e la composizione dell'atto linguistico (Visconti 2009). Tuttavia, nessuna delle ricerche citate si occupa dell'atto linguistico in un corpus italiano, preferendo di gran lunga testi inglesi o slavi.

\section{DESCRIZIONE DEL CORPUS}

Per questo contributo abbiamo costruito un corpus di tre testamenti redatti in italiano da cittadini ragusei nel Trecento e nel Quattrocento. Nell'arco di questi due secoli Ragusa presenta una particolare situazione comunicativa poiché nella città convivono tre lingue: il locale idioma slavo, la variante locale romanza (il raguseo) e il veneziano importato dalla Serenissima (Muljačić 2000; Dotto 2008; Di Salvo 2016). Oltre a queste lingue il latino, essendo la lingua della diplomazia e della scolarizzazione, è ampiamente utilizzato. Insieme alle diverse lingue sono in uso due alfabeti: il latino e il cirillico (Dotto 2008, Đorđić 1990). La lingua ufficiale della cancelleria ragusea è l'italiano perché i cancellieri e i notai che vivevano e lavoravano a Ragusa erano per lo più italiani scolarizzati in Italia (Banfi 2015: 245). I testamenti iniziano a essere redatti in volgare, invece che in latino, in seguito all'epidemia di peste del 1348 (Dotto 2008: 121). 
Per questa ricerca abbiamo selezionato i seguenti testamenti redatti dopo il 1348 in italiano:

1. Il testamento del mercante Milaš Radomirić da Podvisoki, 10/7/1397. (Kurtović et al. 2018: 213-214) - T1

2. Il testamento di Radič Mišetić, 19/6/1449 (Kurtović et al. 2018: 738-742) - T2

3. Il testamento di Pribislav Vukotić, 21/3/1475. (Kurtović et al. 2018: 861-864) - T3

I tre testamenti sono stati trascritti, tradotti e pubblicati per la prima volta nel volume Codex diplomaticus Regni Bosnae (Kurtović et al. 2018). La trascrizione è stata effettuata fedelmente, senza aggiunte o correzioni supplementari. Gli originali dei testamenti sono conservati oggi nell' Archivio di Stato di Dubrovnik, nella raccolta Testamenta Notariae contenente 148 codici e migliaia di testamenti dal 1271 al 1815 in 92 volumi (Lazarević 2016).

\subsection{Struttura del testamento}

Il testamento è uno strumento legale con il quale si legano gli averi alle persone private, alle organizzazioni o agli istituti (Lazarević 2016: 197). Dato che si tratta di un documento legale, il testamento è sempre organizzato secondo una struttura prestabilita e possiede elementi caratteristici e indispensabili. Malgrado il fatto che il testamento possa essere composto da varie formule, esponiamo solo quelle che abitualmente fanno parte del documento. Riprendiamo la struttura del testamento da Stipišić (1985: 148-151), il quale sostiene che, generalmente, ogni testamento è composto da un'introduzione, da un corpo centrale e da una conclusione, elementi che in linguaggio giuridico vengono denominati protocollo, testo o corpus ed escatocollo. Più dettagliatamente, il protocollo è l'introduzione a un atto legale ed è composto dall'invocatio divina (invocazione divina), dall'intitulatio (nome del redattore dell'atto), dall'inscriptio (nome del destinatario) e dalla salutatio (formula di saluto). Poi, il testo o il corpus è composto dall'arenga (un messaggio morale in forma di affermazione che asserisce la buona salute del testatore), dalla dispositio (la parte centrale dell'atto in cui è contenuto il messaggio materiale del testamento) e dalla corroboratio (la formula con cui si assicura l'esecuzione dell'atto legale). L'escatocollo è la parte conclusiva del testamento che contiene la subscriptio (la firma del testatore e dei testimoni) e la datatio (la data e il luogo della redazione dell'atto). Le formule del testamento segnalano che la sua struttura è convenzionale e formulaica. Per quanto concerne questo lavoro e la manifestazione delle formule testamentarie, si ipotizza che le formule si attuino attraverso diversi atti linguistici. 


\section{IL QUADRO DELLA TEORIA TRADIZIONALE DEGLI ATTI LINGUISTICI}

Come già appurato nell'introduzione, il quadro teorico si riferisce teoria tradizionale degli atti linguistici. Tale teoria è stata proposta per la prima volta da John Austin (1962) e poi elaborata più dettagliatamente dal suo allievo John Searle $(1969,1976)$.

Secondo alcuni autori (Austin 1962; Searle 1969, 1976), gli atti linguistici si dividono in atti locutori, illocutori e perlocutori ${ }^{1}$. Gli atti locutori consistono nel dire qualcosa, quelli illocutori nel fare qualcosa dicendo, mentre gli atti perlocutori producono un effetto sull'ascoltatore. Gli atti illocutori sono divisi in cinque classi:

- gli atti verdittivi che rappresentano essenzialmente atti giuridici;

- gli atti esercitivi che rappresentano l'esercizio di potere, di diritti e di influenza;

- gli atti promissivi che obbligano il locutore ad adottare un atteggiamento o effettuare un'azione;

- gli atti comportativi che rappresentano un atteggiamento o una reazione rispetto alla condotta o alla situazione di altri;

- gli atti espositivi che si impiegano per illustrare qualcosa.

Il legame fra la forma grammaticale e il tipo di atto linguistico è già stato esaminato e documentato (Croft 1994; Sadock 1994; Harnish 1994): le caratteristiche sintattiche e morfologiche del verbo possono determinare il tipo di atto linguistico. Secondo Bertucelli Papi (2000), l'uso dell'articolo definito e indefinito, dei dimostrativi, degli affissi, delle forme verbali, dei modi verbali e dell'ordine delle parole può modificare l'interpretazione della forza illocutoria di un enunciato. A titolo esemplificativo, notiamo che i legami intrinsechi fra la forma dell'imperativo e l'atto di ordinare, fra un enunciato interrogativo e una domanda sono evidenti (Escandell Vidal 1996: 64), il che dimostrerebbe l'esistenza di una forma grammaticale tipica per ogni atto linguistico. A ciò si aggiunge che gli atti illocutori sono generalmente riconoscibili grazie all'uso dei verbi performativi (per esempio ordinare, promettere, pronunciare, affermare).

Tuttavia, gli atti linguistici non si manifestano esclusivamente attraverso le strutture grammaticali. A questo proposito Jucker (2000) rileva che l'uso dell'atto linguistico non può essere determinato dalla presenza dei verbi performativi o delle strutture tipiche. Infatti, se osserviamo l'enunciato "Vattene!", l'imperativo rende evidente che si tratta di un ordine e, conseguentemente, di un atto di comando. Ad ogni modo, le situazioni

\footnotetext{
${ }^{1}$ Riprendiamo la terminologia in italiano da Dardano (2005).
} 
comunicative reali raramente sono così semplici e di rado permettono di trascurare il contesto pragmatico dell'enunciato.

Al contesto pragmatico degli atti illocutori si legano due concetti che Austin (1962) definisce come forza illocutoria e come effetto perlocutorio. La forza illocutoria è, infatti, il vero significato dell'enunciato, l'effetto voluto dell'atto, ossia l'intenzione del mittente (Austin 1962: 73). Quanto più esplicito è l'enunciato, tanto la sua forza illocutoria è maggiore. A tale proposito, Austin (1962) distingue tra atti diretti (per esempio l'enunciato "Passami il sale") e indiretti (per esempio "A questo piatto servirebbe un po' più di sale"). L'imperativo è evidentemente esplicito e legato all'atto di comando e la sua forza illocutoria è maggiore di quella del secondo esempio che è invece un comando implicito giacché potrebbe essere interpretato come una constatazione. Da questi due esempi risulta che la struttura grammaticale non coincide necessariamente con la forza illocutoria dell'enunciato.

Per quanto riguarda il concetto di effetto perlocutorio, esso si definisce come l'effetto prodotto da un atto linguistico nell'animo dell'interlocutore. Per esempio, il coraggio è il prodotto dell'atto di incoraggiare, mentre la paura è il prodotto della minaccia. Naturalmente l'appartenenza di un atto linguistico a uno dei gruppi degli atti linguistici è contestuale e interpretativa. Osserviamo la frase "Fa freddo": in base al contesto questa constatazione può essere intesa come un semplice enunciato che prende atto del fatto che la temperatura nella stanza sia più bassa di quella ideale. La situazione comunicativa e il contesto possono essere tali per cui la frase si intende come "Fa freddo, chiudi la finestra". In tal caso, la conseguenza dell'atto può essere, per esempio, che l'interlocutore chiuda la finestra. In questo contesto, 1'enunciato "Fa freddo" è un atto illocutorio poiché ne è conseguita un'azione. In ultimo, sentendo la medesima frase, l'interlocutore può impaurirsi poiché la intende come "Fa freddo, un certo pericolo si sta avvicinando", e così l'enunciato si classifica come atto perlocutorio.

È chiaro che gli atti linguistici non sono schematizzabili, la loro classificazione deve essere attenta e l'effetto perlocutorio non può essere trascurato. Secondo Ariel (2008: 27) i casi in cui si deve concludere sono pragmatici e quelli in cui non bisogna farlo sono grammatici. Dunque, è utilizzando i parametri della grammatica e della pragmatica che definiamo quali atti linguistici vengono utilizzati nei testamenti.

Applichiamo questo quadro teorico nell'analisi e nella determinazione degli atti linguistici nel corpus. Seguendo questa elaborazione teorica, dall'analisi condotta si desume l'uso dei seguenti atti nel corpus: atti locutori (il titolo del testamento e l'elenco dei beni), atti illocutori (nello specifico: atti esercitivi, ossia la dispositio, la nomina dei testimoni e la corroboratio; atti espositivi, ossia l'arenga; e infine gli atti promissivi) e atti perlocutori (le minacce). 


\section{ATTI LOCUTORI}

Come già menzionato nel quadro teorico, gli atti espositivi sono gli atti che trasmettono un'informazione o un messaggio. Data la natura della comunicazione, questi atti sono i più frequenti e li caratterizza la struttura degli enunciati dichiarativi. Il primo atto linguistico che analizziamo, si classifica nel gruppo degli atti espositivi ed è l'inizio dei testamenti: il titolo, ossia l'intitulatio, appartiene alla parte introduttiva del testamento, il protocollo.

I primi due testamenti risultano essere intitolati:

T1 - Testamentum Milassii filii Radomiri (Kurtović et al. 2018: 213) / Il testamento di Milaš, figlio di Radomir;

T2 - Testamentum Radiz Missetich (Kurtović et al. 2018: 738) / Il testamento di Radič Mišetić

La natura giuridica di T1 e di T2 si manifesta con la prima frase redatta poiché viene definito il testatore e si usa esplicitamente la denominazione testamentum (lat. testamento). In ambedue il titolo è composto dal nome in latino seguito dal genitivo del nome del testatore. Il titolo del testamento, infatti, fornisce un'indicazione circa le sorti del documento legale, il che significa che i titoli di T1 e di T2 sono atti locutori, avendo come obiettivo la trasmissione dell'informazione sulla natura del documento, ovvero che l'atto giuridico in questione è un testamento. Di conseguenza il titolo non ha forza illocutoria, dato che svolge solamente una funzione informativa. Considerando che $\mathrm{T} 3$ non è nemmeno intitolato, ne risulta che il titolo non è indispensabile al testamento e non ne inficia il valore legale.

Per quanto riguarda T3, sebbene manchi l'intitolazione in forma di atto espositivo, la natura del testamento è comunque definita dall'atto esercitivo:

T3 - [...] voio che questo sia el mio ultimo testamento. (Kurtović et al. 2018: 861)

È evidente che in T3 si utilizza un verbo performativo (volere) che esprime il desiderio del testatore che l'atto sia un testamento. Dall'uso di questo atto linguistico si evince che $\mathrm{T} 2$ ha la forza linguistica per essere interpretato come un testamento e che infatti, conseguentemente, il testatore agisce usando le parole: egli dichiara l'atto un testamento usando il verbo performativo volere.

Il successivo atto locutorio che si individua nei testamenti è l'elenco dei beni. Si tratta di una semplice elencazione tramite la quale il testatore fornisce informazioni sui beni e sul loro valore monetario. In questa parte del testamento non si accenna a un erede potenziale. Da ciò si può conclu- 
dere che l'elenco dei beni è un atto locutorio perché non viene effettuata nessuna azione, neanche quella del legato dei beni.

A titolo d'esempio si osservino i seguenti due casi da T1 e da T2:

T1 - Agio in debiti come apar scrito per le mie zedule in tuto ducati CXLV. (Kurtović et al. 2018: 214)

T2 - Ago de Stipan Lopatchouich inpegno taza una de argento per ducati et d'oro et yperperi 6 de grossi de Re. (Kurtović et al. 2018: 740)

La struttura dell'elenco è semplice e presenta varianti del presente della prima persona singolare del verbo avere seguito dal complemento oggetto diretto. La natura del testamento come atto personale richiede l'uso della prima persona singolare. Usando questa forma, i testatori avvisano i futuri lettori del testamento quali beni sono a disposizione ma senza compiere nessuna azione. L'elenco dei beni si classifica per questa ragione nel gruppo degli atti locutori. Sebbene l'elenco dei beni appartenga al corpus del testamento, esso non viene considerato parte indispensabile del testamento poiché le ultime volontà del testatore sarebbero espresse anche senza l'elenco. Alla luce di ciò, è evidente che in T3 non è presente alcuna elencazione, e conseguentemente nessun atto locutorio, ma che il testatore prosegue subito con l'atto esercitivo di lasciare i beni agli eredi.

\section{ATTI ILLOCUTORI}

Per quanto riguarda gli atti illocutori presenti nel corpus, è stato possibile individuare gli atti esercitivi, espositivi e promissivi. Gli atti illocutori si sono rivelati il gruppo più numeroso e, perciò, questo capitolo è diviso in tre parti, ciascuna delle quali tratta un diverso tipo d'atto illocutorio.

\subsection{Atti esercitivi}

Il testamento è l'atto esercitivo per eccellenza dato che grazie ad esso si esprime esplicitamente il potere e il diritto di gestire gli averi secondo il proprio volere. Il testatore non deve consegnare personalmente i propri averi all'erede: basta che scriva le sue volontà e che siano eseguite come desidera. Ciò significa che il testatore effettivamente agisce usando le parole e, pertanto, il testamento completo può essere considerato un atto esercitivo. Altre forme del testamento riconoscibili come atti esercitivi sono la dispositio, la corroboratio e la nomina dei testimoni.

La parte centrale del testamento è la dispositio che rappresenta le forme con le quali i beni si legano agli eredi. Considerato che l'obiettivo primario del testamento è il legato degli averi agli eredi, si ritiene che il legato sia di maggior rilievo. Questa ipotesi viene avvalorata anche dall'uso della $d i$ - 
spositio nei testamenti. Nel corpus si individuano varie modalità del legato degli averi, la più tipica delle quali è l'uso dei verbi lasciare e volere grazie ai quali la dispositio è facilmente identificabile.

Nello specifico, i casi sono i seguenti:

T1 - Lasso imprima ala glexia de sancto Nicolo in Bossina a Milli perperi centocinquanta. (Kurtović et al. 2018: 214)

T2 - Item lasso al deto Pauao chauallo negro. (Kurtović et al. 2018: 741)

T3 - Item lasso al dicto Altar un paramento fornado de seda et uno calexe d'arzento [...]. (Kurtović et al. 2018: 861)

Mentre il verbo volere si usa nei contesti che seguono:

T1 - Et lo anunço de li sourascripti denari, voyo che se dia a Pribil Andriasseuich [...]. (Kurtović et al. 2018: 214)

T2 - Item voglio che deueno spender ducati sette per li dableri et candele et altra chose neccessarie [...]. (Kurtović et al. 2018: 741)

T3 - Et ala morte volio la le possi ordenar come li piace. (Kurtović et al. 2018: 862)

I verbi lasciare e volere possiedono lo stesso valore semantico nel contesto poiché il verbo volere non esprime esclusivamente la volontà, ma anche il potere di esercitare. Ciò è spiegabile con il fatto che nel contesto del testamento non è percepibile una differenza essenziale fra l'enunciato "voglio che sia dato" e "io do". La dispositio si classifica nel gruppo degli atti esercitivi perché il testatore è obbligato ad applicare il suo diritto in virtù del proprietario dell'avere per legarlo agli eredi. Conseguentemente, il testatore agisce propriamente usando le parole e adoperando gli atti esercitivi.

Il successivo atto esercitivo effettuato nei testamenti è la corroboratio, ossia la formula con la quale il testatore si assicura l'esecuzione del testamento dopo la sua morte. Abbiamo dimostrato che le formule della corroboratio sono soggette a maggiori variazioni. Una delle modalità di espressione della corroboratio avviene tramite la formula latina:

T1 - Hoc autem testamentum nullo testimonio rumpi possit. (Kurtović et al. 2018: 214) / Che nessuna testimonianza possa rompere questo testamento.

T2 - Quod quidem testamentum nullo testimonio rumpi possit. (Kurtović et al. 2018: 742) / Che questo testamento non possa essere rotto da nessuna testimonianza.

Il verbo possit in ambedue i testamenti è, infatti, il congiuntivo presente del verbo latino posse (it. potere). In questo contesto è usato in senso ortatorio, il che significa che esprime un ordine (Greenough et al. 1903: 278) e si 
classifica nel gruppo degli atti esercitivi grazie all'uso dello specifico modo verbale. Abbiamo appurato che in T3 questo tipo di corroboratio non è stato usato, la sua struttura specifica in T3 sarà esaminata nei capitoli seguenti.

Infine, l'ultima forma appartenente agli atti esercitivi è la nomina dei testimoni. Per assicurare la legittimità del testamento, secondo lo statuto raguseo del 1272, il testamento deve essere redatto in presenza di un notaio e di due testimoni, detti epitropi (Lazarević 2016). La nomina dei testimoni è pertanto un obbligo legale. Tale operazione è riscontrabile, senza eccezioni, in tutti i testamenti del corpus, seppur utilizzando diverse modalità.

I testimoni sono esplicitamente nominati in T1 e T2:

T1 - Lasso li miei pitropi Pribil Andreasseuich e Radin Illich e Radien Tolilouich e Utiessen Bosichouich che li deti possa far e deffar come io [...]. (Kurtović et al. 2018: 214)

T2 - [...] dago liberta alli mey epitropi nominadi de soto. Fazoli mey epitropo frate Vladissao guardian de sancta Maria in Quoyniza. [...] L'altro epitropo Budissauo Slauchouich et Dobrassin Tuedaich deto Vesseochouich e Pauao Radasinouich, chusin mio. (Kurtović et al. 2018: 742)

Si evince che nominare $\mathrm{i}$ testimoni è un tipico atto esercitivo poiché rappresenta l'espressione della volontà del testatore. Sebbene la nomina si riconosca generalmente dall'uso del verbo performativo nominare (Dardano 2005: 178), vengono adoperate anche altre strategie di nomina. Quanto affermato è dimostrato dai casi in T1 e T2. In T1 si utilizza il verbo performativo lasciare: agli epitropi viene lasciato eseguire la volontà del testatore. Da ciò deriva anche la nomina poiché, se il testatore permette ai testimoni il comportamento delineato, li ha, effettivamente, nominati. Nel secondo caso, in T2 si usa l'espressione "dare libertà a qualcuno", seguita dalla specificazione che la libertà è data agli epitropi, il che indica che questa espressione serve a nominare gli epitropi. Questo atto si classifica nel gruppo degli atti esercitivi perché si esercita il potere dell'individuo di nominare il testimone del proprio testamento.

$\mathrm{T} 3$ non segue la formula di T1 e T2, bensì ne presenta una diversa:

T3 - Testis Presbiter Antonius Benedictus Sancti Angeli m.p. / Testis Alexander Clericus Sante Marine m.p. (Kurtović et al. 2018: 864)

È evidente che in T3 i testimoni (in latino testis, termine usato nel testamento) si nominano alla fine del testamento nella formula subscriptio. Questa nomina è implicita, poiché si deve desumere dalla fine del testamento che i due testimoni sono stati presenti durante la stesura del documento. In ogni caso, la subscriptio che nomina due testimoni implica la legalità del testamento. 
Data la natura esercitiva del testamento, era prevedibile che gli atti esercitivi fossero i più numerosi, e ciò si è palesato nel caso del corpus dei testamenti. Si tratta di due formule senza le quali il testamento non è legale: la dispositio e la nomina dei testimoni. A questi due atti si aggiunge anche la corroboratio che si può presentare sotto forma di ordine. Altre modalità della corroboratio saranno analizzate successivamente.

\subsection{Atti espositivi}

Dall'analisi condotta risulta che anche l'arenga si classifica nel gruppo degli atti espositivi. L'arenga è la formula con la quale si esprime che il testatore è in buona salute, sia fisica che mentale, e che è in grado di esercitare il suo diritto di testatore e di ragionare sulle proprie azioni. Giacché l'arenga consta di un'affermazione, ossia di una considerazione individuale che i testatori stabiliscono da sé, e poiché non si citano referti medici o rapporti psichiatrici a conferma della sanità mentale, si deve concludere che l'arenga appartiene al gruppo degli atti espositivi. L'importanza di questa formula è tale che si individua nel corpus intero, espressa con la variazione della stessa affermazione:

T1 - faço lo mio ultimo testamento cum bona mente (Kurtović et al. 2018: 213)

T2 - fazo lo mio ultimo testamento con bona et sana memoria (Kurtović et al. 2018: 738)

T3 - san de la mente per la gratia de Messer domenedio, ma infermo del corpo (Kurtović et al. 2018: 861).

Per determinare l'arenga in un testamento si deve prendere in considerazione il contesto, poiché non vi sono strutture grammaticali che determinino questa formula e l'arenga. Dichiarare la salute psichica non è un'arenga in nessun altro contesto all'infuori di quello testamentario.

\subsection{Atti promissivi}

Le promesse sono il tipico atto promissivo che obbligano l'interlocutore ad adoperare un certo comportamento (Dardano 2005: 178). Le promesse servono al testatore per sincerarsi che, dopo la sua morte, saranno rispettate le sue volontà, il che designa che appartengono alla corroboratio. Solo di rado si incontra una promessa esplicita, cioè espressa con la forma "io prometto" più l'oggetto diretto (Leech 2014: 309-315). Da ciò si conclude che il contesto dev'essere interpretato per poter rilevare la natura della promessa e determinare la forza illocutoria dell'atto. Per illustrare di quale tipo di situazione comunicativa si tratta, si osservino i casi seguenti estrapolati dai testamenti: 
T1 - Et lo anunço de li sourascripti denari, voyo che se dia a Pribil Andriasseuich che lo dicto faça de li ditti denari also albitrio che lo aconça l'anima mia. (Kurtović et al. 2018: 214)

T2 - Et chel deto Pauao sia tegnudo dare a Gruboie Dobrichouich mantello uno de panno de Vizenza [...]. (Kurtović et al. 2018: 741)

T3 - Et chel sacerdote che andara sia obligado a dir messa ogni di a le station de quaresema. (Kurtović et al. 2018: 861)

Questi casi rappresentano particolari situazioni comunicative perché 1'atto di comando implica l'atto di promessa. Oppure, il testatore trasferisce il suo potere al performatore secondario (Kurzon 1986: 39) che è obbligato a promettere. Questi casi non si interpretano come tipici comandi perché, in caso di ordini veri e propri, l'interlocutore è consapevole di non avere il diritto di rifiutare (Leech 2014: 135). D'altronde il testatore può adoperare altri modi per dare disposizioni agli eredi o ai testimoni dall'aldilà, il che implica che deve adoperare altre strategie per assicurare l'esecuzione e l'adempimento della sua volontà. Si tratta infatti di un atto potenziale in quanto il potere delegato al performatore secondario non può essere accertato (Kurzon 1986: 31-40). Dunque, il massimo che il testatore è in grado di compiere è obbligare $\mathrm{i}$ testimoni e gli eredi a promettere di rispettare il testamento.

\section{ATTI PERLOCUTORI}

È difficile determinare gli atti perlocutori perché l'effetto perlocutorio non deve sempre essere chiaro (Jucker 2000) e spesso dobbiamo avere la traccia della risposta o della reazione dell'interlocutore per poter stabilire che un atto linguistico appartiene a questo gruppo. Ogni epoca dispone di proprie regole che sovrintendono al comportamento sociale e alle relazioni intrafamiliari e nel caso della pragmatica storica bisogna inferire dalle motivazioni del mittente del messaggio (Kádár \& Haugh 2013: 161). Alla luce di ciò, le minacce sono l'unico atto perlocutorio individuato nel corpus, anch'esse appartenenti alla corroboratio poiché, come sarà comprovato, servono ad assicurare l'auspicato comportamento degli eredi. Le minacce sono sempre un caso particolare nella produzione degli atti linguistici perché i locutori raramente producono minacce aperte ed esplicite del tipo "io ti sto minacciando" (Blanco Salguiero 2010). Infatti, le minacce sono interpretative, implicite e possono generare malintesi. Ciò implica che si devono intendere secondo la pragmatica e il contesto d'uso. Per quanto riguarda la loro struttura, le minacce sono affini alle promesse: la promessa sottintende un esito positivo per l'interlocutore, mentre il seguito delle minacce è negativo (Blanco Salguiero 2010). Dunque, le minacce nel corpus si individuano 
in base alla conseguenza negativa diretta verso l'interlocutore. Utilizzando questo parametro, l'analisi del corpus ha permesso l'individualizzazione di due minacce:

T1 - Lasso a Stoysaua uxor mia [...] et se vole andar a Ragusa et maritarsi lassoli perperi quatuorcento. Et se la dita andera a Ragusa in monasterio, pregar dio per l'anima mia lassoli ducati seicento. (Kurtović et al. 2018: 214)

T3 - Item se alguna dele dite mie fie se maridasse che Dio non el vora come fano moite senga licentia de la madre in questo caxo non volio habj cosa alguna. (Kurtović et al. 2018: 864)

Risulta evidente dai due casi che le minacce sono espresse con frasi ipotetiche. Tuttavia, non si può affermare l'esistenza di un legame organico fra la forma della frase ipotetica e la minaccia. Per intendere la frase in modo corretto è necessario conoscere il contesto poiché in altre circostanze la frase ipotetica non si interpreta necessariamente come una minaccia, essendo usata primariamente per esprimere una qualsiasi conseguenza. Nel contesto del testamento, l'affermazione in T3 che la figlia non avrà la sua eredità nel caso in cui si sposasse contro la volontà di sua madre è una minaccia, né implicita né tantomeno velata, poiché definisce chiaramente la perdita dell'eredità come esito negativo.

Per quanto riguarda la forma di T1, il fatto che 1'eredità della moglie sarà maggiore s'ella entrasse in monastero si può interpretare come l'espressione di una devozione profonda del marito. Tuttavia, può anche assumere il significato di minaccia, sottintendendo come risultato la diminuzione dell'eredità. In questi casi, le conseguenze negative sono ovvie ed espresse esplicitamente, ossia, l'effetto perlocutorio delle frasi ipotetiche è chiaramente delineato.

È evidente che una proposizione ipotetica non è necessariamente di carattere minaccioso anche dal caso di T2:

T2 - Et se algun delli mey parenti ali quali lasso el mio auer nominado de sopra contradichasse non satisfachare in termine de mexi et da poy la mia morte, che nessun d'essi non possa tor delli mey parenti de quello lassato alli detti. (Kurtović et al. 2018: 742)

Pur essendo una proposizione ipotetica, non implica una conseguenza negativa, bensì solo il desiderio del testatore che nessun parente possa togliere agli altri eredi quello che spetta loro dal testamento. Quindi, anche in questo caso la frase ipotetica rappresenta la corroboratio, seppur in forma di comando.

Da questi esempi emerge l'intenzione del testatore di accertarsi dell'esecuzione testamentaria dopo la morte. Con tutto ciò, è opportuno rimarcare 
che le minacce non sono parte integrante dei documenti legali del Medioevo e che integrarle nel testamento è una decisione individuale del testatore nel tentativo di assicurare il rispetto delle due volontà. Tuttavia, relativamente al fatto che la forma della corroboratio è presente nel corpus nella sua integrità e che il suo contenuto concerne sempre la vita familiare, è possibile ipotizzare che l'individualizzazione degli atti perlocutori nel testamento apra un campo d'indagine sociologica sulla vita nel Quattrocento e, soprattutto, sul ruolo della donna e dei figli.

\section{CONCLUSIONE}

Gli obiettivi di questa ricerca erano diversi: da un lato tentare 1'applicazione degli atti linguistici alla diacronia e dall'altro verificare a che punto la struttura e la natura formulaica del testamento influiscono sulla scelta e sulla natura dell'atto linguistico. I criteri di analisi determinati, il criterio grammaticale e quello pragmatico, si sono mostrati adeguati all'analisi degli atti linguistici nei testamenti e hanno permesso l'applicazione della teoria degli atti linguistici in chiave diacronica.

Tutti gli atti linguistici, indipendentemente dal criterio a cui sono stati sottoposti, provengono dalla struttura del testamento. Si è constatato che ogni formula appartenente al testamento rappresenta un atto linguistico. Gli atti locutori (l'intitulatio, ovvero l'elenco dei beni) non sono indispensabili al testamento e li abbiamo riconosciuti in T1 e T2 grazie alla forma grammaticale.

Poiché il testamento è un atto esercitivo, la sua natura esercitiva si è rivelata dominante. L'analisi ha mostrato che la dispositio, redatta in tutti e tre i testamenti in quanto parte centrale e indispensabile del testamento, la corroboratio e la nomina dei testimoni appartengono al gruppo degli atti esercitivi. La parte più importante della dispositio è il legato dei beni ed è contemporaneamente l'atto più diretto nel corpus, essendo introdotto dai verbi performativi lasciare e volere. I testimoni vengono nominati con varie formule, sia implicite che esplicite, mentre la corroboratio, quando è di natura esercitiva, presenta una variazione della stessa formula latina. Il secondo atto illocutorio individuato è l'arenga, in qualità di atto espositivo, riconoscibile dal contesto e derivante dalla forma del testamento. L'unico atto promissivo nel corpus è quello della promessa. Tuttavia le promesse si trovano al secondo livello di comunicazione e non sono dirette all'interlocutore, appartengono alla corroboratio e obbligano l'interlocutore a un determinato comportamento. L'importanza dell'atto illocutorio nella redazione del testamento è corroborata anche dal fatto che è l'unico attestato, senza eccezioni, nell'intero corpus. 
Quanto agli atti perlocutori, le minacce sono le uniche nel corpus e anch'esse appartengono alla corroboratio perché il loro obiettivo è obbligare gli eredi al rispetto delle ultime volontà del testatore. A proposito della corroboratio, le forme attraverso le quali si esprime questa formula si sono rivelate estremamente varie, mentre gli altri atti sono di carattere fisso e formulaico. Ne consegue che la corroboratio, con le sue manifestazioni nei testamenti medievali, si mostra come un possibile dominio d'indagine su un corpus più ampio.

Pur permanendo certe differenze sul piano formale fra T1 e T2 da un lato e T3 dall'altro, i testamenti del corpus sono redatti secondo la struttura prescritta e tipica del testamento che assicura la loro validità legale. Infine, le formule legali del testamento si realizzano tramite atti linguistici che designano la natura di atto giuridico del documento, per il quale si agisce usando le parole.

\section{BIBLIOGRAFIA}

Ariel, M. (2008). Pragmatics and Grammar. New York: Cambridge University Press.

Arnovick, L. K. (1999). Diachronic Pragmatics. Seven Case Studies in English Illocutionary Development. Amsterdam / Philadelphia: John Benjamins Publishing Company.

Austin, J. L. (1962). How to Do Things with Words. Oxford: Oxford University Press.

Bach, U. (1995). Wills and Will-Making in the 16th and 17th Century England: Some Pragmatic Aspects. In H. A. Jucker (ed.), Historical Pragmatics (pp. 125-144). Amsterdam: John Benjamins Publishing Company.

Banfi, E. (2015). Lingue d'Italia fuori d'Italia. Bologna: il Mulino.

Bartoli, M. G. (2000). Il Dalmatico. Roma: Istituto della Enciclopedia Italiana fondata da Giovanni Treccani S.p.a.

Bertucelli Papi, M. (2000). Is a diachronic speech act theory possible? Journal of Historical Pragmatics, Vol. 1, 57-66.

Blanco Salguiero, A. (2010). Promises, Threats, and the Foundation of Speech Act Theory. Pragmatics 20, 213-228.

Croft, W. (1994). Speech act classification, language typology and cognition. In S. L. Tzohatzidis (ed.), Foundations of Speech Act Theory: Philosophical and Linguistic Perspectives (pp. 460-477). London: Routledge.

Dardano, M. (2005). Nuovo manualetto di linguistica italiana. Zanichelli: Bologna. 
Di Salvo, M. (2016). Fenomeni di convergenza linguistica tra toscano e veneziano in documenti dell'Archivio di Stato di Venezia. In M. Di Salvo \& C. Muru (a cura di), Dragomanni, sovrani e mercanti (pp. 109-146). Pisa: Edizioni ETS.

Dotto, D. (2008). Scriptae venezianeggianti a Ragusa nel XIV secolo. Roma: Viella.

Đorđić, P. (1990). Istorija srpske ćirilice. Beograd: Zavod za udžbenike i nastavna sredstva.

Escandell Vidal, M. V. (1996). Introducción a la pragmática. Barcelona: Ariel Letras.

Greenough, J. B. et al. (1903). New Latin Grammar. Boston: Ginn \& Company.

Harnish, R. (1994). Mood, meaning and speech acts. In S. L. Tzohatzidis (ed.), Foundations of Speech Act Theory: Philosophical and Linguistic Perspectives (pp. 407-459). London: Routledge.

Jacobs, A. \& Jucker, H. A. (1995). The Historical Perspective in Pragmatics. In H. A. Jucker (ed.), Historical Pragmatics (pp. 3-33). Amsterdam: John Benjamins Publishing Company.

Jucker, H. A. (2000). English historical pragmatics: Problems of data and methodology. In G. di Martino \& M. Lima (eds.), English diachronic pragmatics (pp. 17-55). Napoli: CUEN.

Kádár, D. \& Haugh. M. (2013). Understanding Politeness. Cambridge: CUP.

King, J. (2011). Variation through time and text type: The nature of direct and indirect requests in Early Modern Spanish. Spanish in Context 8, $272-294$.

Kohnen, T. (2007). Text types and the methodology of diachronic speech act analysis. In S. M. Fitzmaurice \& I. Taavitsainen (eds.), Methods in Historical Pragmatics (pp. 139-166). Berlin / New York: Mouton de Gruyter.

Kohnen, T. (2008a). Directives in Old English: Beyond Politeness? In A. H. Jucker \& I. Taavitsainen (eds.), Speech Acts in the History of English (pp. 27-44). Amsterdam: John Benjamins Publishing Company.

Kohnen, T. (2008b). Tracing directives through text and time: Towards a methodology of a corpus-based diachronic speech-act analysis. In A. H. Jucker \& I. Taavitsainen (eds.), Speech Acts in the History of English (pp. 295-310). Amsterdam: John Benjamins Publishing Company.

Kryk-Kastovsky, B. (2009). Speech acts in Early Modern English court trials. Journal of Pragmatics 41, 440-457.

Kurtović, E., Dautović Dž., Nakaš, L., Župarić, D. \& Lalić, A. (2018). Codex diplomaticus Regni Bosnae. Sarajevo: Mladinska knjiga. 
Kurzon, D. (1986). It is hereby performed... Explorations in legal speech acts. Amsterdam: John Benjamins Publishing Company.

Lazarević, I. (2016). Nekretnine dubrovačke vlastele u oporukama od 1750. do 1815. godine u Dubrovniku. Povijesni prilozi, Vol. 35, No. $51,197-222$.

Leech, G. (2014). The Pragmatics of Politeness. New York: Oxford University Press.

Lovrić Jović, I. (2006). Fonološka adaptacija talijanizama u dubrovačkim oporukama iz 17. i 18. Stoljeća. Filologija, Vol. 32, No. 1, 173-192.

Lovrić Jović, I. (2013). Dubrovačke ćirilične oporuke iz 17. i 18. stoljeća, Filologija, No. 63, 131-149.

Muljačić, Ż. (2000). Das Dalmatische. Köln - Weimar - Wien: Böhlau Verlag.

Nakaš, L. (2010). Jezik i grafija krajišničkih pisama. Sarajevo: Slavistički komitet.

Sadock, J. M. (1994). Toward a grammatically realistic typology of speech acts. In S. L. Tzohatzidis (ed.), Foundations of Speech Act Theory: Philosophical and Linguistic Perspectives (pp. 393-406). London: Routledge.

Searle, J. R. (1969). Speech Acts: An Essay in the Philosophy of Language. Cambridge: Cambridge University Press.

Searle, J. R. (1976). A Classification of Illocutionary Acts. Language in Society, Vol. 5, 1-23.

Stipišić, J. (1985). Pomoćne povijesne znanosti u teoriji i praksi. Zagreb: Školska knjiga.

Tollerton, L. (2011). Wills and Will-Making in Anglo-Saxon England. Suffolk: York Medieval Press.

Visconti, J. (2009). Speech Acts in Legal Language: Introduction. Journal of Pragmatics, 41, 393-400. 


\section{SPEECH ACT REALIZATION IN MEDIEVAL WILLS}

\section{Summary}

In the present article, we attempt to analyze, through the lens of historical pragmatics, the types of speech acts found in a corpus of Italian wills written in Ragusa during the late fourteenth century and into the fifteenth century. The goals of our research are to determine which speech acts correspond to the form of the will itself, that is, which ones correlate to the structure of the will as a legal document, and which ones are a reflection of wider social influences. In conducting our research, we have taken the theoretical framework provided by Austin (1962) and have tried using it diachronically. The main hypothesis of the research is that most of the speech acts are in keeping with the form of the will and they can be recognized by their grammatical structure. The results of the research imply that Austin's theory may be applied to diachronic research and that the speech acts analyzed for this paper are a result of following the formulaic structure of the will as a legal instrument.

Keywords: wills, legal acts, speech acts, pragmatics, grammar, diachrony. 